\title{
Distribuição de recursos de saúde no Estado de Santa Catarina, Brasil: um subsídio para discussões sobre o financiamento em saúde ${ }^{1}$
}

\author{
Sérgio Fernando Torres de Freitas, ${ }^{2}$ Emil Kupek $^{2}$ \\ e Mayra Chiaradia Perraro ${ }^{3}$
}

RESUMO Objetivo. Identificar a situação de saúde nos municípios de Santa Catarina no ano de 1996 e relacioná-la com gastos efetuados pela esfera federal em saúde em 1997.

Métodos. Foram analisadas, mediante regressão múltipla, a associação entre os recursos federais para atenção à saúde e a mortalidade proporcional, a oferta de serviços de saúde pela rede ambulatorial e hospitalar existente e o tamanho do município; e a associação entre a mortalidade por grandes grupos de causas e a estrutura socioeconômica, a oferta de serviços de saúde pela rede ambulatorial e hospitalar existente e o tamanho de município.

Resultados. A análise de regressão múltipla mostrou associação entre a mortalidade proporcional por: 1) doenças infecto-contagiosas e a mortalidade infantil, o número de profissionais de saúde não-médicos por 10000 habitantes e o número de médicos por 10000 habitantes (associação negativa); 2) doenças crônico-degenerativas e a porcentagem de habitantes com 60 anos ou mais, a mortalidade infantil (associação negativa) e o número de profissionais de saúde não-médicos por 10000 habitantes (associação negativa); e 3) causas externas e o número de habitantes do município, o número de hospitais por 10000 habitantes (associação negativa) e a porcentagem de menores de 1 ano. Os gastos financeiros por habitante em 1997 foram associados principalmente com tamanho do município, número de ambulatórios por 10000 habitantes, indice de mortalidade de Swaroop e Uemura e mortes por doenças crônicodegenerativas no ano de 1996.

Conclusões. Os municípios com melhor perfil de morbimortalidade e estrutura de serviços de saúde receberam mais recursos financeiros federais para saúde. É importante pensar estratégias especiais para garantir aporte adicional de recursos aos municípios com piores indicadores de saúde, para que se consiga reverter esta situação.

Palavras-chave Serviços de saúde, financiamento em saúde, Brasil.

Um dos problemas do planejamento em saúde no Brasil é a pouca quali- dade do monitoramento dos indicadores de saúde, principalmente da mor-

\footnotetext{
Projeto parcialmente financiado pelo Conselho Nacional de Desenvolvimento Científico e Tecnológico $(\mathrm{CNPq})$, através do Programa Institucional de Bolsas de Iniciação Científica (PIBIC).

2 Universidade Federal de Santa Catarina (UFSC), Departamento de Saúde Pública, Florianópolis, SC, Brasil. Correspondência e pedidos de separatas
}

devem ser enviados a Sérgio Torres de Freitas, Departamento de Saúde Pública, CCS, Universidade Federal de Santa Catarina, CEP 88040-900, Florianópolis, SC, Brasil. E-mail: storres@repensul. ufsc.br

3 Universidade Federal de Santa Catarina (UFSC), Curso de Enfermagem. talidade, o que dificulta a localização das áreas mais afetadas, assim como o estabelecimento da relação entre mortalidade e serviços médicos disponíveis. A identificação destes aspectos permitiria redirecionar recursos e atividades aos grupos de maior risco, o que certamente levaria a uma redução dos recursos despendidos. Além disso, 
no contexto das políticas de descentralização e municipalização da atenção primária à saúde, as análises da relação entre situação de saúde e oferta e demanda dos serviços médicos em nível municipal se tornam cada vez mais importantes (1).

No Brasil, essas questões estão diretamente relacionadas ao modelo de financiamento do sistema público de saúde, o Sistema Único de Saúde (SUS). O SUS tem como orientações fundamentais a universalidade, a equiidade e a participação social. Funciona com uma rede própria ou associado aos sistema públicos estaduais e municipais. Além disso, contrata serviços da rede privada sob forma de convênios. Atualmente, é responsável por cerca de $80 \%$ de toda a assistência em saúde prestada no país. Desde sua criação, em 1990, esse sistema tem sido alvo de diversas análises (2-7) que abordam aspectos variados, dentre os quais o repasse de recursos para os municípios e as diretrizes determinantes dos valores destes repasses, de interesse para o presente estudo.

Em 1996 e 1997, grande parte do repasse de verbas federais de saúde para os municípios foi controlado através da norma operacional básica 01/93 (NOB-93), do Ministério da Saúde. Este mecanismo, assim como outros definidos pela Constituição ou por normatizações do Ministério da Saúde, produziu um perfil bem definido de repasse, principalmente se levarmos em consideração que, ao menos no início da década de 1990, os gastos federais compunham a maior parte do total realizado pelo setor público (73\% para o ano de 1990) (8). Tal perfil pode ser sinteticamente caracterizado por: a) vinculação de grande parte dos recursos ao tamanho da população; b) vinculação do pagamento de procedimentos de maior custo à existência de rede prestadora destes serviços e à média histórica de demanda; c) possibilidade de captação de novos recursos através da implantação de programas específicos — os chamados programas verticais - e, posteriormente, do Programa Saúde da Família (PSF) e de projetos ou estu- dos específicos para algumas morbidades; e d) possibilidade de implantação de serviços de vigilância sanitária.

Uma lógica que tem se apresentado com relativa freqüência nesta discussão é a de privilegiar os municípios que tenham maior competência em seus sistemas de saúde. Por exemplo, Ugá (9), ao discutir o papel do Estado enquanto regulador do funcionamento do SUS, propõe um sistema alternativo baseado em um orçamento global ajustado periodicamente segundo a prestação efetiva dos serviços previamente programados e o cumprimento de metas preestabelecidas, itens esses que seriam aliados à autonomia de gestão dos prestadores de serviços. Por outro lado, uma oficina de trabalho promovida em maio de 1995 por diversos órgãos e instituições de saúde (4) propôs que os fundos de participação dos municípios incorporassem em seu cálculo indicadores de carência social e necessidade de serviços com o objetivo de compensar injustiças sociais a partir dos critérios predominantemente populacionais e da alocação de recursos adicionais para os programas de saúde. Essa tendência de se pensar na distribuição de recursos financeiros com base também nos indicadores de saúde e nas necessidades de serviços parece ter sido relegada a segundo plano. Tal fato é compreensível em face da crise continuada do financiamento do setor saúde e da baixa efetividade dos sistemas de saúde municipais, principalmente sob o ponto de vista epidemiológico.

Assim, é importante verificar até que ponto a distribuição dos recursos de saúde está privilegiando áreas com melhores indicadores de saúde. Questões como essas podem ser avaliadas com relativa rapidez e custos mínimos de pesquisa, tempo e recursos humanos utilizando-se o delineamento ecológico.

Recentemente, os estudos ecológicos vêm sendo considerados como um instrumento importante de análise em epidemiologia, principalmente se esta for aplicada ao planejamento em saúde (10). Nos estudos ecológicos, a unidade de análise é a população e não o indi- víduo. Assim, procura-se correlacionar, por exemplo, os dados sobre recursos de saúde em dada cidade com o índice de mortalidade do local. Uma limitação dos estudos ecológicos é que a relação entre as duas variáveis não reflete necessariamente a situação dos indivíduos - ou seja, a existência de associação entre falta de recursos e mortalidade infantil não permitira afirmar que as crianças que morreram por doença tenham sido afetadas pela falta de recursos de saúde (11). Além disso, esse delineamento tem sido criticado em função da chamada falácia ecológica - indevida generalização das características de um agregado, tal como um distrito, para as unidades que o compõem, tais como seus habitantes, bairros ou unidades de saúde (12). Por outro lado, vários trabalhos têm apontado a utilidade e até necessidade dos estudos ecológicos, particularmente no contexto das doenças infecto-contagiosas $(11,13-15)$. Com a crescente informatização e disponibilidade de dados de saúde no Brasil através da Internet (por exemplo, Sistema de Informações Sobre Saúde do Ministério da Saúde, DATASUS) (16), este tipo de análise é um primeiro passo para identificar áreas de prioridade. Outros tipos de estudo devem complementar e aprofundar os achados da análise ecológica, mas seu valor para a definição e reorientação de metas e programas de saúde pública é fundamental.

Assim, o objetivo do presente estudo foi identificar a situação de saúde nos municípios de Santa Catarina no ano de 1996 e relacioná-la com os gastos em saúde efetuados pela esfera federal em 1997 mediante um delineamento ecológico. Mais especificamente, foram analisados dois aspectos: 1) a associação entre recursos federais para atenção à saúde e mortalidade proporcional, oferta de serviços de saúde pela rede ambulatorial e hospitalar existente e tamanho do município; e 2) a associação entre mortalidade por grandes grupos de causas e estrutura socioeconômica, oferta de serviços de saúde pela rede ambulatorial e hospitalar existente e tamanho de município. 


\section{MATERIAIS E MÉTODOS}

Este é um estudo transversal, realizado em 1996 a partir de dados secundários oficiais obtidos em 1999 para o Estado de Santa Catarina através de diversas fontes - Fundação Instituto Brasileiro de Geografia e Estatística (IBGE), DATASUS e Secretaria Estadual da Saúde de Santa Catarina (SESSC), Serviço Nacional de Informação sobre Mortalidade (SIM) e Serviço Nacional de Informação sobre Nascidos Vivos (SINASC) - os dois últimos pertencentes ao Ministério da Saúde adotando-se a totalidade de municípios do Estado (297 municípios) como unidade de análise. Também pode ser caracterizado como um estudo ecológico por utilizar dados agregados por área (município), ao invés de se basear em dados relativos a indivíduos.

A análise estatística usou o método de regressão múltipla para relacionar os desfechos principais com as variáveis independentes. Os recursos financeiros federais do SUS (valores pagos em dezembro de 1996 e em 1997) para cada município foram analisados com relação à mortalidade, dados demográficos e disponibilidade de serviços médicos. A análise da mortalidade incluiu mortalidade por local de residência, mortalidade geral por faixa etária, índice de Swaroop e Uemura (proporção de mortes de pessoas com 50 ou mais anos de idade no total de óbitos), mortalidade por doenças infectocontagiosas, crônico-degenerativas e causas externas e mortalidade infantil padronizada (dados de 1996, exceto mortalidade infantil, dados de 1994 a 1996). Os dados demográficos incluíram distribuição da população por sexo e faixa etária, porcentagem de menores de 1 ano e porcentagem de maiores de 60 anos (dados de 1996, exceto população por sexo e faixa etária, dados de 1994 a 1997). A disponibilidade de serviços médicos levou em conta o número de médicos por 10000 habitantes (dados de 1992), número de profissionais de saúde não-médicos com diploma universitário (PSNM) por 10000 habitantes (1992 a 1996), número de ambulatórios na rede bá-
TABELA 1. Análise de regressão múltipla de fatores associados à mortalidade proporcional por doenças infecto-contagiosas no Estado de Santa Catarina, Brasil, 1996

\begin{tabular}{lrr}
\hline \multicolumn{1}{c}{ Variável independente } & \multicolumn{1}{c}{$\begin{array}{c}\text { Coeficiente } \\
\text { (erro padrão) }\end{array}$} & $P$ \\
\hline $\begin{array}{l}\text { Mortalidade infantil de 1994 a 1996 } \\
\text { Número de profissionais de saúde não médicos com } \\
\quad \text { curso superior por 10 000 habitantes em 1996 }\end{array}$ & $0,00123(0,00041)$ & 0,0032 \\
Número de médicos por 10 000 habitantes em 1992 & $0,00931(0,00264)$ & 0,0005 \\
\hline
\end{tabular}

Fonte: Sistema nacional de informações sobre mortalidade (SIM); sistema nacional de informações sobre nascidos vivos (SINASC); Fundação Instituto Brasileiro de Geografia e Estatística (IBGE); e sistema de informações sobre saúde do Ministério da Saúde (DATASUS).

a Coeficiente não padronizado. sica (1996), número de leitos por tipo (1996), número de hospitais (1996), número de estabelecimentos médicos (1992) e cobertura vacinal para BCG, sarampo, DPT e hepatite B (1996). Cada grupo de mortalidade por causa foi analisado separadamente com relação a serviços médicos, recursos financeiros do SUS e características populacionais.

\section{RESULTADOS}

Os grandes grupos de causas foram analisados separadamente com relação à disponibilidade de serviços médicos, recursos financeiros do SUS e características populacionais. Somente os preditores estatisticamente significativos ao nível de 0,05 para erro tipo 1 serão apresentados.

As doenças infecto-contagiosas foram as causas de morte mais freqüen- tes entre grupos vulneráveis, inclusive menores de 1 ano. Portanto, estão em relação direta com a mortalidade infantil (tabela 1). A tabela 1 mostra também uma associação inversa com o número de médicos, simultaneamente a uma relação direta com número de PSNM por habitante; tal fato indica que a falta de médicos não pôde ser compensada com outros profissionais de saúde quando se trata das doenças infecto-contagiosas e suas complicações.

A relação inversa entre mortalidade proporcional por doenças crônicodegenerativas e mortalidade infantil (tabela 2) indica não somente que estas doenças são as principais causas de morte entre a população adulta e, particularmente, entre os indivíduos com 60 anos ou mais, mas também que são a principal causa de morte em municípios economicamente mais desenvolvidos. Nesse caso, observa-se que,
TABELA 2. Análise de regressão múltipla de fatores associados à mortalidade proporcional por doenças crônico-degenerativas no Estado de Santa Catarina, Brasil, 1996

\begin{tabular}{lcc}
\hline \multicolumn{1}{c}{ Variável independente } & $\begin{array}{c}\text { Coeficiente } \\
\text { (erro padrão) }\end{array}$ & $P$ \\
\hline Mortalidade infantil de 1994 a 1996 & $-0,00112(0,00045)$ & 0,0128 \\
Número de profissionais de saúde não médicos com & $-0,00729(0,00317)$ & 0,0226 \\
$\quad$ curso superior por 10 000 habitantes em 1996 & $0,01611(0,00391)$ & 0,0001 \\
\hline Porcentagem de habitantes com 60 anos ou mais em 1996 &
\end{tabular}

Fonte: Sistema nacional de informações sobre mortalidade (SIM); sistema nacional de informações sobre nascidos vivos (SINASC); Fundação Instituto Brasileiro de Geografia e Estatística (IBGE); e sistema de informações sobre saúde do Ministério da Saúde (DATASUS).

${ }^{a}$ Coeficiente não padronizado. 
TABELA 3. Análise de regressão múltipla de fatores associados à mortalidade proporcional por causas externas no Estado de Santa Catarina, Brasil, 1996

\begin{tabular}{|c|c|c|}
\hline Variável independente & $\begin{array}{l}\text { Coeficientea } \\
\text { (erro padrão) }\end{array}$ & $P$ \\
\hline \multicolumn{3}{|l|}{ População do município em 1996} \\
\hline Mais de 10000 habitantes & $0,02477(0,00835)$ & 0,0033 \\
\hline Até 10000 habitantes & $1,00000^{b}$ & \\
\hline Número de hospitais por 10000 habitantes em 1996 & $-0,01206(0,00495)$ & 0,0219 \\
\hline Porcentagem de menores de 1 ano em 1996 & $0,03366(0,01196)$ & 0,0053 \\
\hline
\end{tabular}

quanto menor o número de PSNM, pior a mortalidade por essas doenças.

Em relação às causas externas (tabela 3), houve associação direta com tamanho da população e proporção de crianças com menos de 1 ano de idade; e inversa com o número de hospitais. Esta observação reflete uma rede hospitalar pequena e de baixa cobertura assistencial; isso ocorre devido à concentração dos hospitais nos municípios de maior contingente populacional, deixando os municípios menores sem atendimento de urgência e ocasionando, assim, uma alta incidência de mortalidade por causas externas.

A tabela 4 indica que os maiores gastos per capita do SUS são aplicados em municípios maiores e com melhor situação de saúde, pois apresentam índice de Swaroop e Uemura mais elevado, maior mortalidade por doenças crônico-degenerativas e melhor capacidade de atendimento na rede básica (pois têm mais postos de saúde e ambulatórios). Levando em consideração que o modelo estatístico adotado ajusta os dados para todas as variáveis, deve-se notar que, mesmo nos municípios pequenos, estes indicadores de mortalidade e morbidade e maior estrutura de atenção primária continuam associados de maneira significativa com o maior recebimento de recursos financeiros para a saúde.

O modelo apresentado na tabela 4 explica $61 \%$ da variância na distribuição dos recursos financeiros federais por municípios, deixando claro que há outros fatores não disponíveis nestes dados que também influenciam a distribuição dos recursos.

\section{DISCUSSÃO}

A discussão teórica sobre distribuição de recursos financeiros para saúde se desenvolve entre dois critérios principais: eqüidade, ou seja, iguais recursos per capita para áreas com mesmas necessidades de saúde, particularmente no caso da atenção primária à saúde; e eficiência técnica dos procedimentos médicos, ou seja, sil, 1997 da Saúde (DATASUS).

a Coeficiente não padronizado.

${ }^{\mathrm{b}}$ Categoria de referência. relação entre recursos utilizados e resultados obtidos, principalmente no caso da rede hospitalar (17).

A avaliação das necessidades de saúde é feita através dos indicadores de mortalidade e morbidade e do nível de rendimento e de cobertura por serviços de saúde. Experiências internacionais em administração de recursos de saúde vêm mostrando a importância de uma regulamentação e fiscalização eficazes dos prestadores de serviços no setor privado (2), cuja tendência é de aumentar a oferta e, assim, o uso de serviços médicos em função do lucro e não necessariamente pelo critério de necessidade. Tal lógica deveria ser estendida tanto para o setor privado quanto para o setor público. Recentes iniciativas do Ministério da Saúde para regulamentar e fiscalizar as seguradoras de saúde e a venda de medicamentos seguem este raciocínio. Além disso, é preciso educar os consumidores, ou seja, vários segmentos da sociedade, sobre a prevenção das doenças e sobre a escolha racional dos serviços de saúde.

No Brasil, a política de financiamento do setor saúde está orientada pelo raciocínio de maior aporte de recursos para municípios com maior e mais complexa capacidade instalada e melhor competência técnica e política

TABELA 4. Análise de regressão múltipla de fatores associados à distribuição dos recursos financeiros do SUS por habitante entre municípios do Estado de Santa Catarina, Bra-

\begin{tabular}{lcc}
\hline \multicolumn{1}{c}{ Variável independente } & $\begin{array}{c}\text { Coeficiente } \\
\text { (erro padrão) }\end{array}$ & $P$ \\
\hline $\begin{array}{l}\text { Índice de Swaroop e Uemura em 1996 } \\
\text { Número de ambulatórios por 10 mil habitantes em 1996 }\end{array}$ & $132,11(35,34)$ & 0,0002 \\
$\begin{array}{l}\text { Porcentagem de mortos por doenças } \\
\quad \text { crônico-degenerativas em 1996 }\end{array}$ & $0,68(0,23)$ & 0,0027 \\
$\begin{array}{l}\text { Tamanho da população do município em 1996 } \\
\quad 5 \text { mil }\end{array}$ & $19,06(8,57)$ & 0,0270 \\
5 a 10 mil & $-56,70(5,74)$ & 0,0001 \\
10 a 50 mil & $-49,44(5,52)$ & 0,0001 \\
50 a 100 mil & $-42,19(5,35)$ & 0,0001 \\
$>100$ mil & $-32,01(5,91)$ & 0,0001 \\
\hline
\end{tabular}

Fonte: Sistema nacional de informações sobre mortalidade (SIM); sistema nacional de informações sobre nascidos vivos (SINASC); Fundação Instituto Brasileiro de Geografia e Estatística (IBGE); e sistema de informações sobre saúde do Ministério 
para a captação de recursos através de mecanismos ordinários de incentivo. Acontece também que os municípios que possuem estas condições, em muitos casos - como demonstrado para o Estado de Santa Catarina - também são os que apresentam melhores indicadores de saúde e socioeconômicos.

A concentração dos recursos nos centros de referência das cidades com mais de 100 mil habitantes talvez seja eficaz para tratamentos de saúde que necessitam de alta tecnologia, mas não para a prevenção, onde a rede básica tem mais peso. $\mathrm{O}$ uso de valor agregado financeiro dos recursos disponíveis per capita para cada município não permite quantificar diretamente o peso do setor hospitalar nesta distribuição, sendo isso uma limitação deste estudo, pois os gastos de municípios maiores poderiam ser mais altos devido à existência de uma rede de alta complexidade também maior. Por outro lado, os indicadores de oferta de serviços de saúde, tais como número de médicos e PSNM por habitante, medem indiretamente o desenvolvimento do setor hospitalar e ajustam parcialmente o efeito deste fator na distribuição dos recursos do SUS.

Os recursos financeiros do SUS têm sido cada vez melhor controlados, fazendo diminuir o temor de que aportes financeiros em municípios pequenos e mal estruturados fossem mal empregados. No entanto, este maior controle financeiro e a política de distribuição destes recursos não têm garantido a eqüidade do sistema, embora seja notório o aumento da transparência dos gastos com saúde.

A montagem de estratégias especiais de financiamento de projetos para diminuição de mortalidade, a redefinição de algum percentual dos orçamentos federal e estadual e a melhor maneira de investimento destes recursos em municípios com os piores indicadores de saúde se tornam então uma pauta importante para discussão e redirecionamento do financiamento público para a saúde no Brasil, principalmente se adotados sob uma ótica de transitoriedade; ou seja, a adoção destes programas e recursos específicos para municípios com piores perfis de morbimortalidade e estrutura de saúde não deve, nem pode, perpetuar estas condições.

\section{REFERÊNCIAS}

1. Paim JS. Políticas de descentralização e atenção primária à saúde. Em: Rouquayrol $\mathrm{MZ}, \mathrm{Al}-$ meida Filho N, eds. Epidemiologia e saúde. Rio de Janeiro: MEDSI; 1999. pp. 489-504.

2. Campos AC. Normativismo e incentivos: contribuição da economia para a administração da saúde. Em: Piola SF, Vianna SM, eds. Economia da saúde: conceito e contribuição para a gestão da saúde. Brasília: Instituto de Pesquisa Econômica Aplicada (IPEA); 1995. pp. 69-98.

3. Carvalho G. A implantação do sistema único de saúde: nós críticos a nível estadual. Saúde em Debate (Londrina) 1994;45:4-8.

4. O financiamento do SUS - oficina de trabalho promovida pelo CONASS, CONASEMS, MS, OPAS/OMS, ABRES E IDISA [relatório]. Saúde em Debate (Londrina) 1995;47: 67-69.

5. Ugá MAD. Instrumentos de avaliação econômica dos serviços de saúde: alcances e limitações. Em: Piola SF, Vianna SM, eds. Economia da saúde: conceito e contribuição para a gestão da saúde. Brasília: Instituto de Pesquisa Econômica Aplicada (IPEA); 1995. pp. 209-226.
6. Silva MGC. Economia da saúde. Em: Rouquayrol MZ, Almeida Filho N, eds. Epidemiologia e saúde. Rio de Janeiro: MEDSI; 1999. pp. 457-472.

7. Porto SM. Justiça social, eqüidade e necessidade em saúde. Em: Piola SF, Vianna SM, eds. Economia da saúde: conceito e contribuição para a gestão da saúde. Brasília: Instituto de Pesquisa Econômica Aplicada (IPEA); 1995. pp. 123-140.

8. Medici AC. Aspectos teóricos e conceituais do financiamento das políticas de saúde. Em: Piola SF, Vianna SM, eds. Economia da saúde: conceito e contribuição para a gestão da saúde. Brasília: Instituto de Pesquisa Econômica Aplicada (IPEA); 1995. pp. 23-68.

9. Ugá MAD. Competição administrativa pelo estado: notas para discussão. Saúde em Debate (Londrina) 1997;46:14-18.

10. Almeida Filho N, Rouquayrol MZ. Introdução à epidemiologia moderna. Rio de Janeiro: COOPMED/Associação Brasileira de Pós-Graduação em Saúde; 1989.

11. Susser M. The logic in ecological: I. The logic of analysis. Am J Public Health 1994;84(5): 825-829.
12. Morgenstern $\mathrm{H}$. Uses of ecologic analysis in epidemiological research. Am J Public Health 1982;72(12):1336-1344.

13. Koopman JS. The ecological effects of individual exposures and nonlinear disease dynamics in populations. Am J Public Health 1994;84(5):836-842.

14. Schwartz S. The fallacy of the ecological fallacy: the potential misuse of a concept and the consequences. Am J Public Health 1994;84(5): 819-824.

15. Susser M. The logic in ecological: II. The logic of design. Am J Public Health 1994;84(5):830835.

16. http://www.datasus.gov.br [site da Internet]. Acessado em 16 de julho de 2001.

17. Giraldes MR. Distribuição de recursos num sistema público de saúde. Em: Piola SF, Vianna SM, eds. Economia da saúde: conceito e contribuição para a gestão da saúde. Brasília: Instituto de Pesquisa Econômica Aplicada (IPEA); 1995. pp. 167-190.

Manuscrito recebido em 10 de outubro de 2000. Aceito em versão revisada em 31 de maio de 2001. 
ABSTRACT Objective. To describe the health situation in municipalities in the state of Santa Catarina, Brazil, in 1996, and to investigate how that correlated with federal health spending in 1997.

\section{The allocation of health care resources in the state of Santa Catarina, Brazil: a contribution to a discussion on health funding}

Methods. Multiple regression analysis was used to investigate the association between federal health care funding and proportional mortality, supply of health services (hospitals and outpatient clinics), and the municipality's population (number of inhabitants). Also investigated was the association between mortality from broad groups of causes and socioeconomic structure, supply of health services, and the municipality's population.

Results. The multiple regression analysis showed an association between proportional mortality due to: 1) infectious diseases and: infant mortality, number of nondoctor medical professionals per 10000 inhabitants, and number of physicians per 10000 inhabitants (negative association); 2) chronic degenerative diseases and: percentage of individuals 60 years and older, infant mortality (negative association), and number of non-doctor medical professionals per 10000 inhabitants (negative association); and 3) external causes of death and: the municipality's population, number of hospitals per 10000 inhabitants (negative association), and percentage of children younger than 1 year. Health spending per inhabitant in 1997 was mainly associated with the municipality's population, number of outpatient clinics per 10000 inhabitants, Swaroop and Uemura mortality rate, and deaths due to chronic degenerative diseases in 1996.

Conclusions. Municipalities with a better morbidity and mortality profile and a better health services structure received more federal health care resources. To improve this situation, special strategies should be considered in order to ensure additional resources for municipalities that have poorer health indicators.

All scientific investigations are faced with the problem of eliminating unknown bias from test and control observations. Where, as in the present case, the findings are based on human data and involve comparisons between facts which have been obtained by different observers from inexpert witnesses, the main sources of such bias are: 1) unequal recording of events by different observers; 2) unequal reporting of events by the individuals chosen to represent case and control groups; and 3) faulty selection of cases and/or controls.

[Todas las investigaciones científicas encierran el problema de eliminar sesgos desconocidos de las observaciones efectuadas en pacientes y testigos. En casos como este, en que los resultados se basan en datos obtenidos de seres humanos y encierran comparaciones entre datos recogidos por distintos observadores de sujetos inexpertos, las principales fuentes de estos sesgos son las siguientes: 1) el registro desigual de los fenómenos por distintos observadores; 2) la notificación desigual de los fenómenos por las personas elegidas para representar a los pacientes y testigos; y 3) la selección inadecuada de los casos, los testigos, o ambos.]

Alice Stewart, Josephine Webb, \& David Hewitt "A survey of childhood malignancies" British Medical Journal, 1958; 